\title{
Malposition épididymaire et oligospermie
}

\author{
R. SCHOYSMAN
}

Hôpital Van Helmont, Vilvorde, Belgique

\section{RESUME}

Au cours d'explorations détaillées de patients souffant d'oligospermie sévère il a été mis en évidence un certain nombre de situations au cours desquelles le testicule présente une spermatogénèse normale contrastant nettement avec les éjaculats répétitivement très médiocres et présentant en outre des anomalies de position des épididymes. Ces anomalies étaient bilatérales et impliquaient la disparition totale de la queue épididymaire.

Une explication physiopathologique des conséquences de ces anomalies sur la qualité du sperme éjaculé peut-être déduite à partir des travaux expérimentaux au cours desquels des créations de situations cryptépididymaires ont abouti à des oligospermies similaires.

Mots clés : épididyme, spermatogénèse, oligospermie.

\section{INTRODUCTION}

\section{Justification de la biopsie testiculai-} re

Dans le cadre de l'exploration du patient sévèrement oligospermique, la biopsie testiculaire est tombée en disgrâce dans de très nombreux centres. Cette évolution est compréhensible étant donné que le résultat d'une biopsie n'implique pratiquement jamais des conséquences thérapeutiques bien définies. Notre approche de la biopsie testiculaire aurait connu la même évolution, si ce n'est que la collaboration avec une équipe génétique ne nous avait incité à faire des prélèvements testiculaires pour rechercher des anomalies méiotiques [7]. Il est alors apparu qu'en cas d'oligo-asthéno-tératospermie sévère un certain nombre d'anomalies génétiques de la méiose pouvaient être décelées, qui seraient sinon passées inaperçues, les caryotypes périphériques restant normaux [8].

Mais l'usage relativement fréquent de biopsie testiculaire pour oligospermie a permis de mettre en évidence un autre groupe d'anomalies : celles où la spermatogénèse s'avéra parfaitement normale mais où l'épididyme était induré et porteur de séquelles inflammatoires qui avaient mené à des degrés variables de subobstruction. Nous en avons dégagé le concept de l'oligospermie épididymaire [12-14]. L'oligospermie épididymaire est loin d'être exceptionnelle. Les indications de la biopsie testiculaire dépendent tant de l'examen clinique que du bilan hormonal du patient. A l'examen clinique on palpe des testicules normaux et souvent des têtes épididymaires légèrement indurées. Le bilan hormonal comporte les examens de routine, de LH, FSH, prolactine et testostérone. Les éjaculats sont étudiés selon les critères stricts actuellement en usage et sont retenus de préférence les cas où il y a moins de $14 \%$ de formes normales dans les frottis. 
2. Détails de l'exploration de 2000 cas (en 30 ans) d'oligospermie sévère c'està-dire moins de $5 \mathrm{M} / \mathrm{ml}$., moins de $20 \%$ de motilité et moins de $25 \%$ (14\% depuis 1 an) de formes normales [11]

L'analyse de 100 biopsies testiculaires chez des hommes sévèrement oligospermiques a permis la distribution suivante :

- spermatogénèse déficiente accompagnée d'anomalies méiotiques $17 \%$

- spermatogénèse normale avec épididyme pathologique

$49 \%$

- Spermatogénèse déficiente avec méiose normale $24 \%$

- Spermatogénese normale et épididymes normaux $10 \%$

L'importance du facteur épididymaire dans le domaine de l'oligospermie ressort clairement de ce tableau. Pratiquement dans la moitié des cas les causes de la déficience spermatique ne sont pas localisées au niveau de la spermatogénèse.

\section{SITUATIONS CLINIQUES PARTICULIERES}

Parmi les 2000 explorations scrotales chez des hommes sévèrement oligospermiques nous avons trouvé 9 patients chez lesquels il $\mathrm{y}$ avait des situations anatomiques très inhabituelles de l'épididyme. Parmi eux, 4 avaient une inversion simple de la situation anatomique normale, c'est-à-dire que la tête épididymaire se trouvait au pôle inférieur du testicule et qu'au niveau de la queue de l'organe on ne ressentait pas l'épaississement habituellement palpable dans cette région (Figure 1). Une situation un peu différente a été trouvée dans trois autres cas dans lesquels la tête épididymaire est également au pôle inférieur mais après $+/-15$ $\mathrm{mm}$. l'organe se sépare tout à fait du testicule et continue vers le cordon de façon rectiligne et sans qu'on puisse encore y palper une induration correspondant à la queue épididymaire (Figure 2). Un cas montrait une particularité très inhabituelle : la tête était attachée au pôle supérieur du testicule sur 12 à $13 \mathrm{~mm}$. et puis l'épididyme se recourbe immédiatement vers le haut en continuation directe avec le canal déférent (Figure 3).

Enfin, dans un cas l'attache de la tête épididymaire était inhabituellement courte, +/$10 \mathrm{~mm}$., et le corps était en continuité directe avec le canal déférent (Figure 4).

La situation était bilatérale dans tous les cas. Tant à l'inspection qu'à la palpation les têtes épididymaires paraissaient normales sans zones indurées ou bleuâtres, ni hypervascularisées. Nous pensions que l'oligospermie sévère était la conséquence de lésions localisées plus profondément dans l'organe ou ne touchant qu'une très petite partie du tractus.

Il est important de noter que ces patients ne présentaient pas d'autres problèmes andrologiques. Toute recherche de varicocèle même subclinique était négative. Les bilans hormonaux sont dans des limites parfaitement normales: L'oligospermie dont ils étaient porteurs avait été observée à de très nombreuses reprises et pendant des délais forts longs, leur infertilité ayant une durée moyenne de plus de 4 ans. Les biopsies testiculaires ont été lues selon deux scores : notre lecture quantitative habituelle [14], voisine du score de Johnsen ainsi gue le score selon Silber et Rodriguez-Rigau [13]. Si une corrélation mathématique précise de la lecture de biopsie testiculaire selon Silber et Rodriguez Rigau est parfois mise en doute, il n'empêche que c'est une indication quand même assez intéressante pour l'interprétation des éjaculats.

\section{DISCUSSION}

En dehors de la malposition épididymaire observée il n'y avait aucune zone d'induration qui permettait de classer ces cas dans les oligospermies épididymaires liées à des difficultés de transit dans cet organe et nous n'avons pas d'explication rationnelle à avancer pour expliquer l'oligospermie 


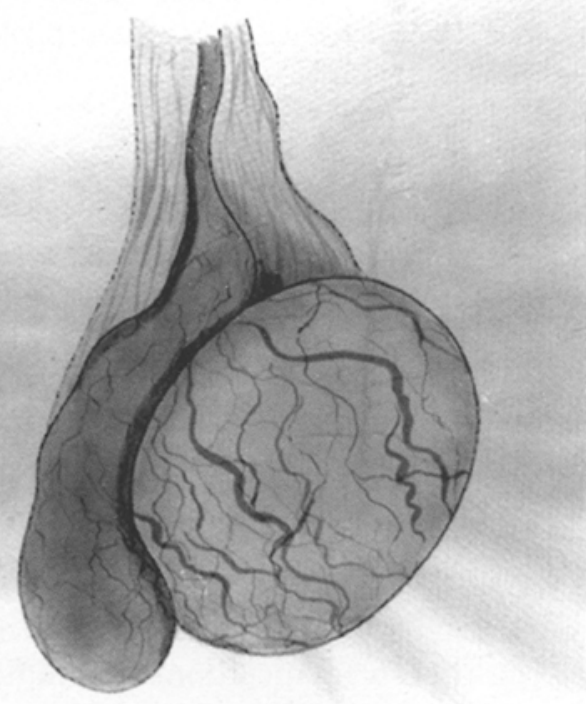

Figure 1 : Inversion totale de l'épididyme dont la partie caudale est encore à peine palpable.

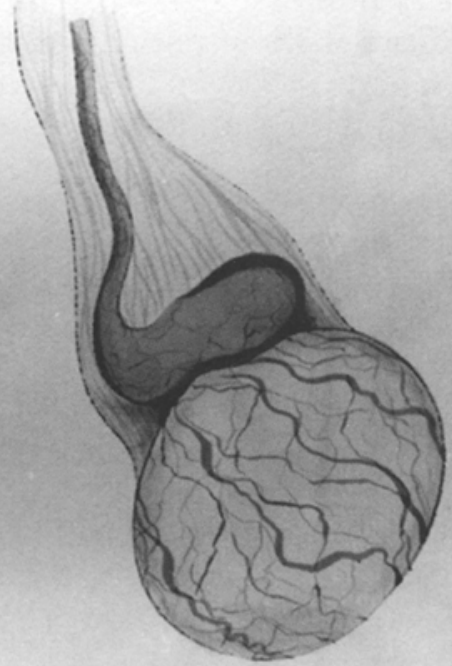

Figure 3 : Position normale de la tête épididymaire mais incurvation du corps de l'organe vers le haut et disparition de la queue.

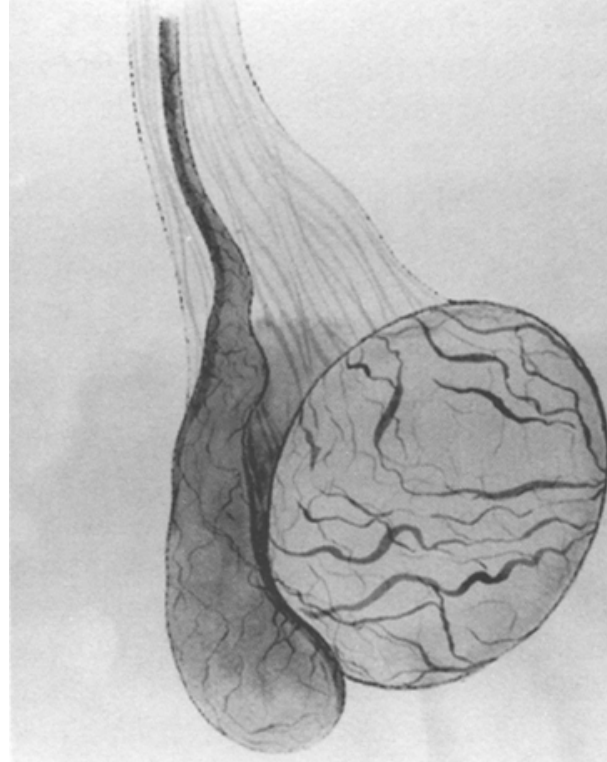

Figure 2 : Inversion totale de l'épididyme avec séparation du testicule à partir des canaux efférents et disparition de la queue.

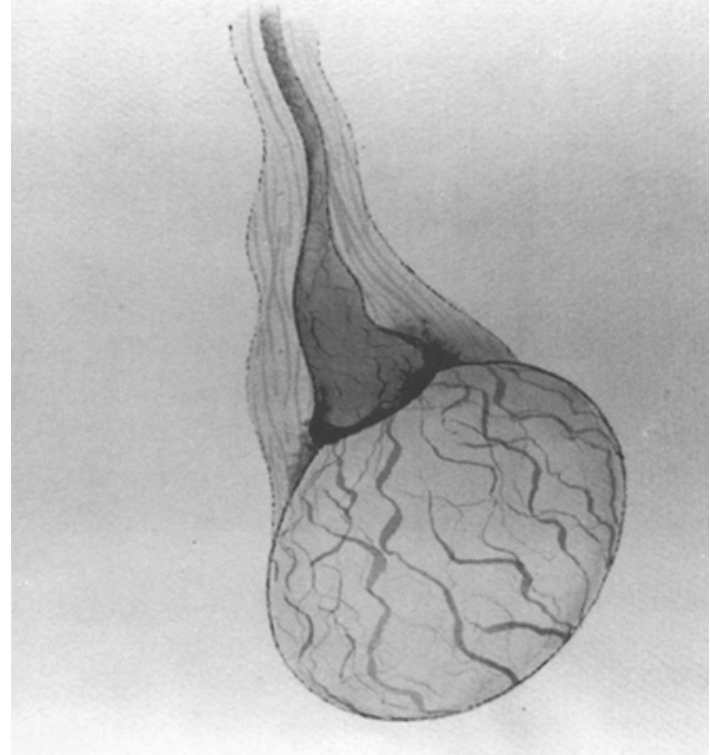

Figure 4 : Structure très inhabituelle de l'épididyme avec prolongement direct des canaux efférents vers le haut dans le cordon. 
constatée. D'autant moins qu'il manque évidemment une série témoin d'hommes à fertilité connue. Mais des travaux expérimentaux réalisés par Bedford [1-2] chez le rat ont fait revoir ce problème. Cet auteur a bien démontré que la création de situations cryptépididymaires ne menait pas à des difficultés de transit mais à d'autres anomalies fonctionnelles du tube épididymaire. Il a réalisé diverses expérimentations afin de mieux comprendre l'effet de variation de température sur la fonction de l'épididyme. Elles mènent à la conclusion que la possibilité de la queue épididymaire de maintenir les spermatozoïdes en un état viable est rapidement éliminée à température corporelle. La création d'une situation "cryptépididymaire" peut d'ailleurs, après recorrection chirurgicale, être parfaitement réversible et la conclusion de tous ces travaux est que la queue de l'épididyme est un organe thermosensible et qu'une température de 37 entraîne rapidement l'altération de sa fonction avec des conséquences dans la composition de son contenu sécrétoire. Dans ces conditions, malgré une production testiculaire constante la numération spermatique dans l'éjaculat est très sévèrement diminuée. Il serait erroné de transposer des résultats chez le rat directement à des situations cliniques humaines. Chez l'homme, la queue de l'épididyme est certainement moins développée que chez le rat où il s'agit d'un organe bien dilaté au pôle inférieur du testicule. Néanmoins, le problème de la température scrotale élevée a toujours préoccupé les andrologues. Ces élévations de température sont toujours interprétées comme causes de déficience testiculaire mais il n'y a pas de preuve convaincante pour prouver cette affirmation. Dès lors, reste la suspicion que la capacité de réservoir de la queue épididymaire humaine pourrait être supprimée par des variations de température scrotale. Seule l'exploration soigneuse de mâles présentant une O.A.T. peut argumenter le débat. Malheureusement, la biopsie testiculaire se pratique de moins en moins quoique la compréhension de situations andrologiques soit importante ne fut-ce que pour éviter que des patients soient traités pendant des délais déraisonnables par des thérapeutiques prolongées, coûteuses et inutiles.

\section{CONCLUSIONS}

L'oligo-asthéno-tératospermie est un syndrome présentant de nombreux aspects. La maladie épididymaire y occupe une place importante et, dans notre expérience, est responsable d'environ $40 \%$ des déficiences séminologiques alors qu'on retient généralement en premier lieu les lésions testiculaires. Chez des patients oligospermiques l'exploration permet d'isoler un groupe dans lesquel un épididyme anatomiquement sain pourrait interférer avec la qualité du sperme de par sa malposition. Ces mâles, porteurs d'une excellente spermatogénèse, mais d'une oligospermie très sévère, présentent une situation anatomique similaire à celle créée expérimentalement chez des rongeurs chez lesquels des situations cryptépididymaires ont des conséquences très importantes sur la qualité du sperme éjaculé. Cette similitude entre l'expérimentation chez le rat et les situations cliniques rapportées n'est pas pour autant une preuve formelle que la malposition épididymaire est cause de sévère oligospermie. Cette preuve pourrait être apportée par une amélioration du sperme après restitution anatomique normale (comme expérimentalement chez le rat), mais le raccourcissement congénital des épididymes dans les situations décrites ne permit pas cette tentative. Cette pathologie andrologique étant rare il serait dès lors intéressant de rassembler davantage de dossiers les mettant en évidence pour progresser dans une meilleure compréhension de ce type particulier de dysfonction épididymaire.

\section{REFERENCES}

1. BEDFORD J.M. : Maturation, transport and fate of spermatozoa in the epididymis in GREEP R.O. Handbook of physiology. Section 7 Vol. 5 : 303-317. 
2. BEDFORD J.M. : Effects of elevated temperature in the epididymis and testis : experimental studies. In "Temperature and environmental effects in the testis" Ed. Zorgniotti, Plenum Press - NY 1991.

3. BLAQUIER J.A., CAMEO M.S., CUASNICU P.S., GONZALES ECHEVERRIA M.F., PINEIRO L., TEZON S.G., VAZQUEZ M.H. : On the role of epididymal factors in sperm fertility. Reprod. Nutr. Develop. 1988, 28.(5) 1209.

4. COUROT M. : Transport and maturation of spermatozoa. Progress Reprod. Biol. 1981, 8:67.

5. FOURNIER-DELPECH S., THIBAULT CH. : Maturation epididymaire, glandes annexes et capacitation. in 'La Reproduction chez les mammifêres et l'homme. INRA 1991.

6. GLOVER T.D. : Spermatozoa from the isolated cauda epididymis of rabbits and some effects of artificial cryptorchidism. J. Reprod. Fertil. 1, 121.

7. KOULISCHER L., SCHOYSMAN R. : Chromosomes and human infertility. Mitotic and Meiotic chromosome studies in 202 male infertile patients. Clinical Genetics. 1974, 5, 116.

8. KOULISCHER L., SCHOYSMAN R. : Etude des chromosomes mitotiques et meiotiques chez les hommes infertiles. C.R. 3ème Journées Européennes de Conseil Génétique. Médecine et Hygiène, Genève 1975, 58.

9. ORGEBIN-CRIST M.C., FOURNIER-DELPECH S. : Sperm-egg interaction. Evidence for maturational changes during epididymal transit. J. Androl. 1982, 3, 429.

10. SCHOYSMAN R. : Epididymal causes of male infertility. Int. Congress Fertility, Miami 1975.

11. SCHOYSMAN R. : Epididymal causes of male infertility : pathogenesis and management. Int. J. Androl. 1982, Suppl. 5, 120.
12. SCHOYSMAN R. : L'oligospermie épididymaire Reprod. Nutr. Develop. 1988, 28.(5) 1339

13. SILBER S.J., RODRIGUEZ-RIGOU L.S. : Quantitative analysis of testicle biopsy : determination of partial obstruction and prediction of sperm count after surgery for obstruction. Fertil. Steril. 36, 480.

14. VAN DE CASSEYE M., SCHOYSMAN R. : Pathologie de l'épididyme et répercussion sur la fonction testiculaire. Reprod. Nutr. Dévelop. 1988, 28.(5) 1347.

\section{ABSTRACT \\ Malposition of the epididymis and oligospermia}

\section{R. SCHOYSMaN}

Hôpital Van Helmont, Vilvorde, Belgique

In the course of investigation of men suffering from severe oligospermia the author has met with cases were spermatogenesis was normal in testicular biopsies, contrasting with repeatedly severe oligospermia, the situation being accompanied by an obvious malposition of the epididymis. The main anomaly of the epididymis was the absence of its habitual caudal structure. There is no clear physiopathological explanation for biopsy/sperm discrepancy linked to epididymal malposition, but experimental work concerning cryptepididymal situations may offer a clue.

Key-words : spermatogenesis, epididymis, oligospermia. 